INPLASY

PROTOCOL

To cite: Luo et al. The aim of this meta-analysis of randomized controlled trials is to evaluate the efficacy and safety of rituximab for frequently relapsing nephrotic syndrome in children. Inplasy protocol 2021110099. doi: 10.37766/inplasy2021.11.0099

Received: 28 November 2021

Published: 28 November 2021

Corresponding author: Ningxin Luo

zhaoli@scmc.com.cn

Author Affiliation:

Shanghai Children's Medical Center.

Support: None.

Review Stage at time of this submission: Preliminary searches.

Conflicts of interest: None declared.

\section{The aim of this meta-analysis of randomized controlled trials is to evaluate the efficacy and safety of rituximab for frequently relapsing nephrotic syndrome in children}

Luo, N1; Shen, X²; Zhao, L33.

Review question / Objective: Rituximab, a chimeric anti-CD20 monoclonal antibody, is a novel drug used in the treatment of various autoimmune diseases. The aim of this meta-analysis of randomized controlled trials is to evaluate the efficacy and safety of rituximab for frequently relapsing nephrotic syndrome in children.

Eligibility criteria: (1) Children with frequently relapsing nephrotic syndrome who were younger than 18 years' old; (2) The studies assessed the efficacy and safety of Rituximab for treating frequently relapsing nephrotic syndrome; (3) The studies compared rituximab with placebo or other conventional drugs; (4) The study's design was randomized controlled trial.

INPLASY registration number: This protocol was registered with the International Platform of Registered Systematic Review and Meta-Analysis Protocols (INPLASY) on 28 November 2021 and was last updated on 28 November 2021 (registration number INPLASY2021110099).

\section{INTRODUCTION}

Review question / Objective: Rituximab, a chimeric anti-CD20 monoclonal antibody, is a novel drug used in the treatment of various autoimmune diseases. The aim of this meta-analysis of randomized controlled trials is to evaluate the efficacy and safety of rituximab for frequently relapsing nephrotic syndrome in children.

Condition being studied: Idiopathic nephrotic syndrome is the most common chronic glomerular disease in children, occurring in two to seven of 100,000 children per year. At least $20 \%$ of children with this syndrome show frequent relapses 
and/or steroid dependence during or after immunosuppressive therapies, a condition defined as complicated frequently relapsing/steroid-dependent nephrotic syndrome (FRNS/SDNS). Standard treatments worldwide for FRNS/SDNS in children are immunosuppressive agents, including cyclophosphamide, chlorambucil, cyclosporine (CyA), tacrolimus, and levamisole, whereas the standard treatment for SRNS in children is CyA. However, some patients may endure a complicated clinical course or even develop end stage renal failure. Rituximab, a chimeric anti-CD20 monoclonal antibody, is a novel drug used in the treatment of various autoimmune diseases. The aim of this meta-analysis of randomized controlled trials is to evaluate the efficacy and safety of rituximab for frequently relapsing nephrotic syndrome in children. Cyclophosphamide, calcineurininhibitor, mycophenolate mofetil, and alkylating agents, or various combinations of these drugs are the most commonly used steroid sparing protocols in SRNS and SDNS; however, most of these drugs not only present serious adverse effects, but also so many cases cannot achieve complete remission and will develop end stage renal failure. Rituximab is a novel drug which we will try to evaluate by our meta-analysis.

\section{METHODS}

Search strategy: ("Nephrotic Syndrome"[MeSH Terms] OR "nephrotic syndromes"[Title/Abstract] OR "syndrome nephrotic"[Title/Abstract] OR "syndromes nephrotic"[Title/Abstract]) AND ("Rituximab"[MeSH Terms] OR "cd20 antibody rituximab"[Title/Abstract] OR "rituximab cd20 antibody"[Title/Abstract] OR "Mabthera"[Title/Abstract] OR "idec c2b8 antibody"[Title/Abstract] OR "idec c2b8 antibody"[Title/Abstract] OR "IDECC2B8"[Title/Abstract] OR "GP2013"[Title/ Abstract] OR "Rituxan"[Title/Abstract]) AND ("Child"[MeSH Terms] OR "Children"[Title/Abstract] OR "Infants"[ Title/Abstract] OR "Adolescents"[Title/Abstract] OR "Adolescence"[Title/Abstract] OR "Teens"[Title/Abstract] OR "Teen"[Title/
Abstract] OR "Teenagers"[Title/Abstract] OR "Teenager"[Title/Abstract] OR "Youth"[Title/Abstract] OR "Youths"[Title/ Abstract] OR "adolescents female"[Title/ Abstract] OR "adolescent female"[Title/ Abstract] OR "female adolescent"[Title/ Abstract] OR "female adolescents"[Title/ Abstract] OR "adolescents male"[Title/ Abstract] OR "adolescent male"[Title/ Abstract] OR "male adolescent"[Title/ Abstract] OR "male adolescents"[Title/ Abstract] OR "Adolescent"[MeSH Terms] OR "Infant"[MeSH Terms]).

Participant or population: Children with FRNS who were younger than 18 years old will be included.

Intervention: Receiving any dose of Rituximab.

Comparator: Placebo or other receiving conventional drugs.

Study designs to be included: RCT.

Eligibility criteria: (1) Children with frequently relapsing nephrotic syndrome who were younger than 18 years' old; (2) The studies assessed the efficacy and safety of Rituximab for treating frequently relapsing nephrotic syndrome; (3) The studies compared rituximab with placebo or other conventional drugs; (4) The study's design was randomized controlled trial.

Information sources: PubMed, Web of Science, Cochrane library.

Main outcome(s): Relapse-free survival rate.

Additional outcome(s): The Relapse Numbers; proteinuria excretion; serum albumin; serum creatinine.

Data management: Two authors (NL, XS) will independently extract data. Any disagreement will be resolved by discussion until consensus is reached or by consulting a third author. The following data will be extracted: first author, publication year, countries, total numbers and dropout of subjects, age, duration of 
intervention, dosage, relapse-free survival rate, biological indictors, adverse effects.

Quality assessment / Risk of bias analysis: The risk of bias was evaluated by the Cochrane bias risk assessment tool of RevMan software. Two reviewers (NL, XS) will independently assess risk of bias based on the following domains from recommendations from the Cochrane handbook: 1. Adequate sequence generation; 2. Allocation concealment; 3. Blinding; 4. Incomplete outcome data and how it was addressed; 5 . Selective reporting of the outcome; 6. Any other biases. results of bias assessment will be presented in a figure and a graph indicating low, high or unclear risk of bias for each of the 6 items in each trial. Disagreements between individual judgements will be resolved by discussion and consensus.

Strategy of data synthesis: We will use the Rev Man 5.3 software to process the data. 12 and Chi 2 tests will be used to estimate heterogeneity. When $12>50 \%$, the randomeffect model will be used; otherwise, a fixed effects model will be utilized to process data. Subgroup analysis might be conducted to identify the possible sources of heterogeneity.

Subgroup analysis: Not planned yet. Subgroup analysis might be conducted to identify the possible sources of heterogeneity.

Sensitivity analysis: After deleting any one of the study, the results of the other studies were not significantly different from the results before deletion, indicating the sensitivity analysis has been done appropriatly.

Language: English.

Country(ies) involved: China.

Keywords: Rituximab; Refractory nephrotic syndrome; Meta-analysis; Efficacy; Safety.

Contributions of each author:

Author 1 - Ningxin Luo.

Email: Inx1993@qq.com
Author 2 - Xiaoyu Shen.

Email: shenxiaoyu@scmc.com.cn

Author 3 - Li Zhao.

Email: zhaoli@scmc.com.cn 